\title{
REVIEW
}

\section{Pomalidomide: the new immunomodulatory agent for the treatment of multiple myeloma}

\author{
AA Chanan-Khan ${ }^{1}$, A Swaika ${ }^{1,2}$, A Paulus $^{1}$, SK Kumar ${ }^{3}$, JR Mikhael ${ }^{4}$, SV Rajkumar ${ }^{3}$, A Dispenzieri ${ }^{3}$ and MQ Lacy ${ }^{3}$
}

\begin{abstract}
In this report, we provide a comprehensive review on the preclinical and clinical investigations conducted in development of the next-generation immunomodulatory drug (IMiD) pomalidomide for the treatment of relapsed/refractory multiple myeloma (MM). We consulted PubMed, MEDLINE, ASH, ASCO annual symposium abstracts and http://clinicaltrials.gov/ for the purpose of this literature review. Twenty-six preclinical and 11 clinical studies were examined. These studies delineate the mechanisms of action of pomalidomide and attest to the robust clinical activity in relapsed/refractory MM. MM is the second most common hematological malignancy in the US. Despite availability of several therapeutic agents, MM remains incurable. Thus, the development of new therapies remains a priority. Pomalidomide is the newest member of the IMiDs class of drugs, and in preclinical and clinical investigations, it has demonstrated an improved efficacy and toxicity profile in comparison to its sister compounds, lenalidomide and thalidomide. Importantly, recent clinical studies have demonstrated its activity in relapsed or refractory myeloma, particularly in lenalidomide and bortezomib-refractory patients. Thus, the addition of pomalidomide to the anti-myeloma armamentarium is widely anticipated to have a significant impact on the overall clinical outcome of advanced stage relapsed and refractory MM patients.
\end{abstract}

Blood Cancer Journal (2013) 3, e143; doi:10.1038/bcj.2013.38; published online 6 September 2013

Keywords: multiple myeloma; immunomodulatory agents; pomalidomide; treatment

\section{INTRODUCTION}

Immunomodulatory drugs (IMiD) are a novel class of orally available antineoplastic agents that have made an important impact on the treatment of patients with hematological malignancies, particularly multiple myeloma (MM). The parent compound, thalidomide, was the first to demonstrate antineoplastic activity among patients with $\mathrm{MM}^{1}{ }^{1}$ with subsequent investigations conclusively establishing its anticancer effects. This fueled further research into the development of its more potent and less toxic analogs. In order to increase the immunomodulatory capabilities, and thus antitumor activity of the thalidomide analogs, the next IMiD, lenalidomide, was formulated with a substitution of an amino group at the fourth position and a single oxo group in the phthaloyl ring. ${ }^{2}$ Consistent with the thalidomide experience, lenalidomide also delivered impressive clinical responses among patients with malignant disorders. While the major impact of the IMiDs is noted among patients with MM, notable clinical benefit has also been established in several other hematologic cancers including myelodysplastic syndrome, non-Hodgkin lymphoma and chronic lymphocytic leukemia. ${ }^{3,4}$

Pomalidomide (previously known as CC-4047, actimid) is the most recent and potent IMiD molecule, and its steady clinical development is widely anticipated to redefine the therapeutic landscape for relapsed and refractory MM patients. Pomalidomide differs from thalidomide by the addition of two oxo groups in the phthaloyl ring and an amino group at the fourth position (Figure 1), ${ }^{5,6}$ This comprehensive review will focus on the clinical experience with pomalidomide in MM. We will also summarize the seminal preclinical investigational efforts made in understanding the mechanisms by which this exciting new IMiD molecule imparts its clinical activity.

\section{PRECLINICAL EVALUATION OF POMALIDOMIDE}

The exact molecular mechanisms of the antitumor effects of pomalidomide remain uncertain. As a group, IMiDs broadly affect various molecular and cellular elements within the tumor microenvironment, affecting the concentration of various cytokines that support tumor cell growth (Figure 2). ${ }^{7}$ IMiDs are known to suppress the production of tumor necrosis factor-alpha (TNF- $\alpha$ ), interleukin-12 (IL-12), IL-16, IL-1 $\beta$, macrophage inflammatory protein-1 alpha (MIP-1 $\alpha$ and monocyte chemoattractant protein-1 (MCP-1), while increasing the yield of IL-2, IFN- $\gamma, \mathrm{IL}-10$ and RANTES (regulated upon activation, normal T-cell expressed, and secreted) by LPS-induced peripheral blood mononuclear cells (PBMCs). ${ }^{8}$ The increased production of IFN- $\gamma$ and IL-2 results from the direct co-stimulation of T-cells. ${ }^{7}$ It is postulated that the enhanced activity of protein kinase C-theta (PKC- $\theta$ ) and activated protein-1 (AP-1) in these stimulated T-cells increases IL-2 levels, leading to an amplified NK-cell response. ${ }^{9-11}$ Participation of the innate immune system, by NK-cell activation, is a key component in mounting a successful antitumor response, regardless of whether pomalidomide acts on NK cells directly or indirectly. As such, pomalidomide enhances NK-cell cytotoxicity and antibody-dependent cell-mediated cytotoxicity (ADCC) in MM cells. ${ }^{12}$ This effect has been observed in other hematological malignancies, such as Burkitt's lymphoma, where pomalidomide was administered with rituximab, and an increased survival rate

\footnotetext{
${ }^{1}$ Department of Hematology and Oncology, Mayo Clinic, Jacksonville, FL, USA; ${ }^{2}$ Department of Internal Medicine, State University of New York at Buffalo, Buffalo, NY, USA; ${ }^{3}$ Department of Hematology and Oncology, Mayo Clinic, Rochester, MN, USA and ${ }^{4}$ Department of Hematology and Oncology, Mayo Clinic, Scottsdale, AZ, USA. Correspondence: Dr AA Chanan-Khan, Department of Hematology and Oncology, Mayo Clinic, 4500 San Pablo Road, Jacksonville, FL 32224, USA.

E-mail: chanan-khan.asher@mayo.edu

Received 15 July 2013; accepted 17 July 2013
} 
<smiles>O=C1CC[C@H](N2C(=O)c3ccccc3C2=O)C(=O)N1</smiles>

Thalidomide<smiles>Nc1cccc2c1CN(C1CCC(=O)NC1=O)C2</smiles>

Lenalidomide

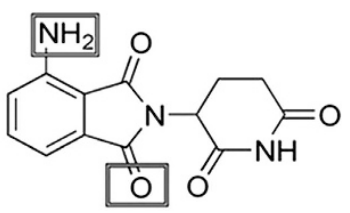

Pomalidomide

Figure 1. Chemical structure of the IMiD drugs.

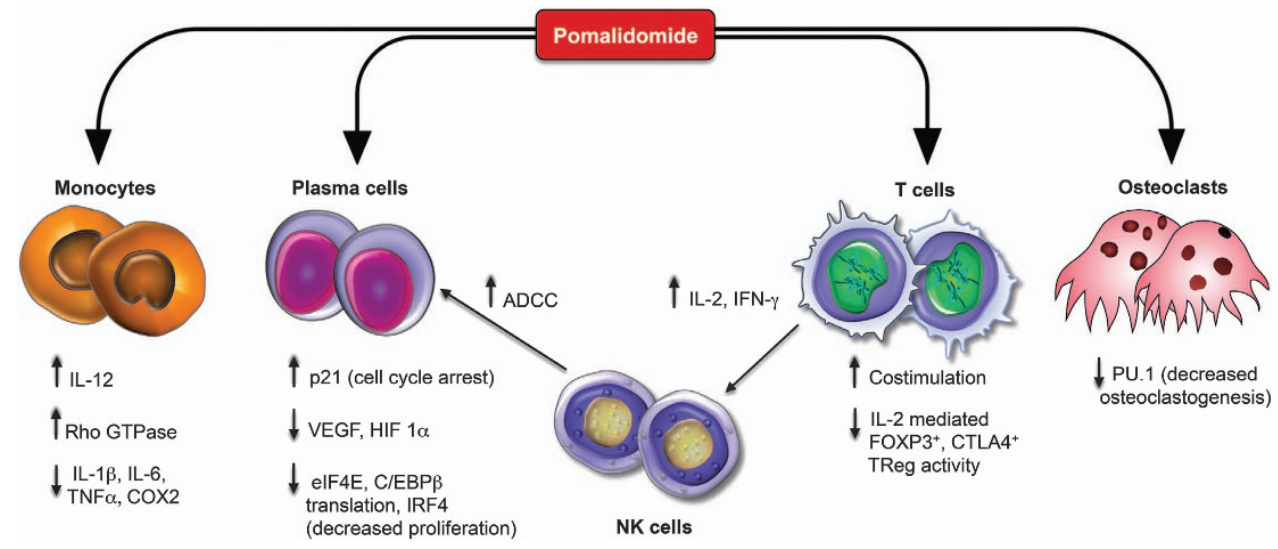

Figure 2. Activity of pomalidomide in MM.

was observed among mice treated with this combination therapy. However, this benefit was abrogated upon depletion of NK-cells, thus establishing the role of NK cells in an IMiDs-induced tumor response. ${ }^{13}$ Dredge et al, $^{14}$ using pomalidomide as a priming agent in a murine tumor cell vaccination model of ectopic colorectal cancer, further explored the abilities of a pomalidomide-mediated immune-enhancement. An 80\% survival rate was observed among mice given pomalidomide in conjunction with the CT26 tumor vaccine. ${ }^{14}$ This conferred protection was found to be long lasting and associated with the propagation of a tumor-specific Th-1 type response with increased production of IFN- $\gamma$. Another possible mechanism for this adjuvant-like tumor-specific response generated by treatment with pomalidomide might be due to the inhibition of IL-2mediated production of FOXP3 + , cytotoxic T-lymphocyte Antigen $4(\mathrm{CTLA}-4+), \mathrm{CD} 4+\mathrm{T}$ regulatory cells. ${ }^{15}$ Most studies have examined the role of IMiDs in the context of co-stimulatory molecules; however, $\mathrm{Xu}$ et al. $^{16}$ demonstrated that the primary mechanisms of pomalidomide modulation remain in its ability to alter intracellular structures such as the actin cytoskeleton and microtubules via upregulating the Rho family of GTPases.

In addition to modulation of lymphoid-derived cells in the tumor milieu, pomalidomide acts on osteoclasts $(\mathrm{OCL})$, which are responsible for lytic bone disease in MM. OCLs arise from cells of the monocyte/macrophage lineage and various cytokines such as granulocyte macrophage colony-stimulating factor (GM-CSF), IL-3 and receptor activator of nuclear factor kappa-B Ligand (RANKL) control their differentiation. The transcription factors, c-fos and PU.1 have crucial roles in osteoclastogenesis, with the latter being downregulated by lenalidomide and pomalidomide, resulting in the inhibition of bone resorption. ${ }^{17,18}$

Neo-angiogenesis is a hallmark of tumor growth, and in MM it is interceded by the stromal cells of the microenvironment in response to vascular endothelial growth factor (VEGF), which is secreted by MM cells. Pomalidomide inhibits stromal cell adhesion ${ }^{19}$ and has been shown to markedly inhibit angiogenesis by decreasing concentrations of VEGF and hypoxia-inducible factor 1 alpha $(\mathrm{HIF}-1 \alpha)^{20,21}$

MM patients have been shown to express high levels of the inflammatory molecule cycolooxygenase-2 (COX-2), which is associated with poor disease outcome. ${ }^{22}$ In a multicenter Phase II trial, Prince et al. ${ }^{23}$ demonstrated improvement in progressionfree and overall survival (PFS and OS, respectively) when a highdose COX-2 inhibitor (celecoxib) and thalidomide were given to patients with advanced MM. Consistent with other IMiDs, pomalidomide exerts anti-inflammatory effects by inhibition of transcriptional activity of the COX-2 gene, thereby reducing COX-2 and prostaglandin levels. ${ }^{24}$

Both in vitro and in vivo investigations attest to the direct anti-MM activity of pomalidomide. Pomalidomide induces cell cycle arrest in both lymphoma and MM cells, independent of p53 signaling, via a Lysine-specific demethylase 1 (LSD1)-mediated epigenetic mechanism, resulting in increased expression of p21-WAF. ${ }^{25}$ Pro-apoptotic activity has been noted in MM cells treated with pomalidomide, resulting in caspase-8 induction, suppression of nuclear factor kappa-B (NF- $\mathrm{kB}$ ) transcription, cellular inhibition of IAP-2 (inhibitor of apoptosis 2) and increased sensitivity to Fas-mediated cell death. ${ }^{26} \mathrm{MM}$ cells rely heavily on various transcription factors such as IL-6, interferon regulatory factor 4 (IRF4) and B-lymphocyte-induced maturation protein 1 (BLIMP1) to sustain their proliferative capacity. Through blockade of CAAT-enhancer-binding protein beta (C/EBP $\beta$ ) translation in MM cell lines, pomalidomide is able to reduce downstream IRF4 expression, leading to the inhibition of malignant growth. Interestingly, overexpression of C/EBP $\beta$ in these cells leads to an abrogation of pomalidomide induced anti-proliferative effects. ${ }^{27}$

The reduction in IRF4 levels has proven to be mediated by a direct effect of IMiDs on the target cell protein cereblon (CRBN). CRBN is the primary target and binding partner identified for the teratogenic effects of thalidomide and interacts with the DNA damage binding protein-1 (DDB1) to form a functional E3 ligase 
complex with the Cul4A and Roc $1 .{ }^{28}$ More recently, lenalidomide and pomalidomide have been demonstrated to target CRBN. ${ }^{29}$ This complex possesses autoubiquitlating activity that is inhibited in the presence of IMiDs and is in part responsible for its teratogenic effects. Furthermore, CRBN has been shown to interact with IRF4 and SPIB, two transcription factors critical for myeloma (and $A B C$ non-Hodgkin lymphoma) cell survival and whose downregulation was directly linked to synthetic CRBN depletion with a resultant loss of lenalidomide-mediated antitumor activity. ${ }^{30}$ Zhu et al. ${ }^{31,32}$ recently demonstrated that CRBN is not only essential for the antitumor activity of the IMiDs but also its depletion is associated with IMiD resistance. Notably, CRBN expression does not affect the antitumor activity of other key anti-MM agents. Recent data establishes a positive correlation between pre-treatment CRBN expression levels and response to treatment with pomalidomide plus dexamethasone in $M M$ patients. $^{33}$ Measurement of CRBN expression in MM patients carries potential utility as a biomarker that is predictive of therapeutic response to pomalidomide (or possibly any IMiD). As such, optimal use of the anti-MM effects of pomalidomide can be focused towards the appropriate patient population in which CRBN-associated activity can be fully exploited. It is interesting to note that pomalidomide demonstrates robust efficacy in patients who are resistant/refractory to lenalidomide. Perceptibly, there are multiple distinct reasons for IMiDs resistance and its inter-class variability.

\section{CLINICAL EVALUATION OF POMALIDOMIDE IN MM}

The clinical efficacy of pomalidomide has been investigated in phase I-III clinical trials and the pertinent details are comprehensively outlined in Table 1 . Schey et al., ${ }^{34}$ who examined its safety and tolerability in relapsed refractory MM patients in a phase I clinical trial, initially carried out a formal study of pomalidomide. The main objective of the study was to establish the maximumtolerated dose (MTD) and safety of pomalidomide with 24 patients enrolling in the trial. The median age of the enrolled patients was 66 years, and all patients had previously received a median of three lines of therapy (range, 1-6). The doses of pomalidomide investigated were $1,2,5$ or $10 \mathrm{mg}$. The median duration of study treatment for all patients was 28 weeks (range, 3-132 weeks). All 24 patients were eligible for response assessment and the overall response rate (ORR) was $54 \%$, with $17 \%$ of patients achieving a

\begin{tabular}{|c|c|c|c|c|c|c|c|c|}
\hline Trial & Phase & $\mathrm{N}$ & $\begin{array}{l}\text { Median } \\
\text { age (years) }\end{array}$ & $\begin{array}{l}\text { Median } \\
\text { prior Tx }\end{array}$ & Regimen & Dose schedule/MTD & $\begin{array}{l}\text { ORR } \\
\text { (\%) }\end{array}$ & PFS; OS (months) \\
\hline Schey et al. ${ }^{34}$ & I & 24 & 66 & 3 & $\begin{array}{l}\text { Pom dose } \\
\text { escalation }\end{array}$ & $\mathrm{MTD}=2 \mathrm{mg} / \mathrm{day}$ & 54 & $9.7 ; 22.5$ \\
\hline Streetly et $a l^{35}$ & I & 20 & 58 & 4 & Pom $+I-$ Dex & $\mathrm{MTD}=5 \mathrm{mg}$ q.o.d & 50 & $10.5 ; 33$ \\
\hline \multirow[t]{3}{*}{ Richardson et al. ${ }^{41}$} & & 113 & & 5 & Pom/Dex & $\mathrm{MTD}=4 \mathrm{mg}^{\mathrm{a}} ; 40 \mathrm{mg}^{\mathrm{b}}$ & 34 & $4.6 ; 14.4$ \\
\hline & $1 / I I$ & & 63 & & & & & \\
\hline & & 108 & & 5 & Pom & $4 \mathrm{mg}^{\mathrm{a}}$ & 13 & $2.6 ; 13.6$ \\
\hline Lacy et al. ${ }^{36}$ & $\|$ & 60 & 66.5 & 2 & Pom/Dex & $2 \mathrm{mg}^{\mathrm{c}} ; 40 \mathrm{mg}^{\mathrm{b}}$ & 63 & $11.6 ; 76 \%$ at 2 years \\
\hline Lacy et al. ${ }^{37, d}$ & II & 34 & 62 & 4 & Pom/Dex & $2 \mathrm{mg}^{c} ; 40 \mathrm{mg}^{\mathrm{b}}$ & 47 & $4.8 ; 13.9$ \\
\hline \multicolumn{9}{|l|}{ Leleu et al. ${ }^{43}$} \\
\hline Arm A & $\|$ & 43 & 54 & 4 & Pom/Dex & $4 \mathrm{mg}^{\mathrm{a}} ; 40 \mathrm{mg}^{\mathrm{b}}$ & 30 & NA \\
\hline Arm B & & 40 & & 4 & & $4 \mathrm{mg}^{\mathrm{c}} ; 40 \mathrm{mg}^{\mathrm{b}}$ & 47 & NA \\
\hline \multicolumn{9}{|l|}{ Lacy et al. ${ }^{39, \mathrm{e}}$} \\
\hline Cohort A & $\|$ & 35 & & 6 & Pom/Dex & $2 \mathrm{mg}^{\mathrm{c}} ; 40 \mathrm{mg}^{\mathrm{b}}$ & 49 & $6.5 ; 78 \%$ at 6 months \\
\hline Cohort B & & 35 & & 6 & & $4 \mathrm{mg}^{\mathrm{c}} ; 40 \mathrm{mg}^{\mathrm{b}}$ & 43 & $3.2 ; 67 \%$ at 6 months \\
\hline \multicolumn{9}{|l|}{ Dimopoulos et al. ${ }^{45}$} \\
\hline Cohort A & III & 302 & NA & 5 & Pom/Dex & $4 \mathrm{mg}^{\mathrm{a}} ; 40 \mathrm{mg}^{\mathrm{b}}$ & 21 & 3.6; NR \\
\hline Cohort B & & 153 & NA & & Dex & $40 \mathrm{mg}^{f}$ & 3 & 1.8; NR \\
\hline \multicolumn{9}{|l|}{ Shah et al. ${ }^{46,9}$} \\
\hline & I/II & 32 & 61 & 6 & $\begin{array}{l}\text { Carf } \\
\text { Pom } \\
\text { Dex }\end{array}$ & $\begin{array}{c}20 / 27 \text { and } 20 / 36 \mathrm{mg} / \mathrm{m}^{2, \mathrm{~h}} \\
4 \mathrm{mg}^{\mathrm{a}} \\
40 \mathrm{mg}^{\mathrm{b}}\end{array}$ & 33 & NA \\
\hline \multicolumn{9}{|l|}{ Rossi et al. ${ }^{47,9}$} \\
\hline & $1 / I I$ & 66 & NA & 3 & $\begin{array}{l}\text { Clar } \\
\text { Pom } \\
\text { Dex }\end{array}$ & $\begin{array}{c}500 \mathrm{mg} \text { b.i.d } \\
4 \mathrm{mg}^{\mathrm{a}} \\
40 \mathrm{mg}^{\mathrm{b}}\end{array}$ & 56 & $5, \mathrm{NA}$ \\
\hline \multicolumn{9}{|l|}{ Palumbo et al. ${ }^{48,9}$} \\
\hline & $1 / I I$ & 52 & 69 & 3 & Pom & $1-2.5 \mathrm{mg}^{\mathrm{c}}$ & 73 & $\begin{array}{c}52 \% \text { at } 1 \text { year; } 78 \% \text { at } \\
1 \text { year }\end{array}$ \\
\hline & & & & & Cyclo & $50 \mathrm{mg}$ q.a.d & & \\
\hline & & & & & Pred & $50 \mathrm{mg}$ q.a.d & & \\
\hline
\end{tabular}

Abbreviations: Carf, carfilzomib; Clar, clarithromycin; Cyclo, cyclophosphamide; Dex, dexamethasone; Median prior Tx, Median number of prior treatments; MTD, maximum-tolerated dose; NA, not available; NR, not reached; ORR, overall response rate; OS, overall survival; Pom, pomalidomide; PFS, progression-free survival; Pred, prednisone; q.a.d, every other week; q.o.d, every other day. ${ }^{\mathrm{a}}$ Days $1-21$ of a 28 -day cycle; ${ }^{\mathrm{b}}$ Days $1,8,15,22$ of a 28 -day cycle, ${ }^{\mathrm{c} D a y s ~} 1-28$ of a 28-day cycle; ${ }^{\mathrm{d}}$ Study was conducted in lenalidomide-refractory patients ${ }^{\mathrm{e}}$ Study was conducted in lenalidomide and bortezomib-refractory patients; ${ }^{\mathrm{f}}$ Days $1-4$,

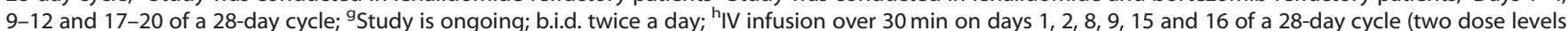
tested in this study). 
complete remission (CR). The MTD was defined as $2 \mathrm{mg}$ and the main dose-limiting toxicity (DLT) was grade 4 neutropenia.

Streetly et al. $^{35}$ also conducted a Phase I clinical trial with 20 patients receiving alternate day pomalidomide dosed at 1, 2, 5 and $10 \mathrm{mg}$. Pomalidomide was continued beyond 4 weeks among patients with no clinical or biochemical evidence of disease progression, provided they had recovered from any grade three or four toxicities. The major toxicity reported in this clinical trial was grade 4 neutropenia, and the $5 \mathrm{mg}$ alternate dose was assigned as the MTD. The ORR and CR were $50 \%$ and $10 \%$, respectively. In nine patients, dexamethasone (20 mg alternate day $1-4,15-18)$ was added because of progressive disease, which induced a clinical response in four out of nine patients. The median PFS was 10.5 months (19.7 months for responding patients) and the median OS was 33 months. Investigators concluded that the MTD of singleagent pomalidomide in patients with relapsed/refractory MM was $2 \mathrm{mg}$ administered as a daily dose and $5 \mathrm{mg}$ for the alternate dose schedule.

These trials laid the foundation for subsequent clinical investigation. Lacy et al. ${ }^{36}$ conducted a Phase II trial of pomalidomide in combination with dexamethasone among patients who had received less than three prior therapies. Sixty patients were given pomalidomide ( $2 \mathrm{mg}$ daily) with dexamethasone $40 \mathrm{mg}$ weekly. The median age of the patients enrolled on this clinical trial was 65.5 years. Forty percent of the patients had lenalidomide-refractory disease. The ORR was $63 \%$ with $5 \%$ of the patients achieving CR. The most common grade 3 or 4 toxicity was neutropenia.

Based on the highly encouraging clinical responses exhibited with pomalidomide therapy in patients with lenalidomideresistant disease, Lacy et al. ${ }^{37}$ conducted another Phase II study specifically focusing on patients with lenalidomide-refractory MM. The primary goal of this therapy was to assess the ORR and the duration of response to pomalidomide-based therapy. Pomalidomide was given as $2 \mathrm{mg}$ daily dose, day 1 through 28 on a 4-week cycle. Forty milligrams of dexamethasone was given concurrently on days 1, 8, 15 and 22. Patients were also given aspirin for prophylaxis of any venous thrombo-embolic events. The study design allowed for an increase in the dose of pomalidomide to $4 \mathrm{mg}$, if patients failed to respond to two cycles of $2 \mathrm{mg}$ pomalidomide. Thirty-four patients were enrolled in this clinical trial with a median age of 61.5 (range 39-77) years and had previously received a median of four lines of therapy. The ORR noted was $47 \%$ with the common toxicity being grade 3 or 4 neutropenia (26\%). Of the 34 patients enrolled, 19 had previously received thalidomide, and 20 patients had received prior therapy with bortezomib. Notably, in this subset of patients, a clinical response was observed among 42 and $45 \%$ of patients with prior exposure to thalidomide or bortezomib, respectively.

Patients who have relapsed and/or are refractory to both bortezomib and an IMiD (dual refractory disease) carry a poor clinical outcome. ${ }^{38}$ In a dual refractory MM patient population, Lacy et $a l^{39}$ evaluated the impact of pomalidomide with the primary objective of determining ORR and duration of response to two different doses of pomalidomide. Patients were divided into two cohorts of 35 each. Patients in cohort 1 received a $2 \mathrm{mg}$ dose of pomalidomide, and those in cohort 2 received a $4 \mathrm{mg}$ dose of pomalidomide daily on days 1-28 with dexamethasone given on days $1,8,15$ and 22 of a 28-day cycle. The dose of pomalidomide could be increased to $4 \mathrm{mg} /$ day if patients failed to demonstrate a clinical response or developed disease progression. The ORR among patients in the $2 \mathrm{mg}$ cohort was $49 \%$ as compared with $43 \%$ in the $4 \mathrm{mg}$ cohort. Both cohorts reported an almost similar number of patients with stable disease. The results of this study indicated that pomalidomide was highly effective in patients with dual refractory MM; however, there was no additional benefit in clinical efficacy upon administration of a higher dose of pomalidomide.
Richardson et al. ${ }^{40}$ also reported results of a Phase I/II multicenter clinical trial investigating the role of pomalidomide alone or in combination with low-dose dexamethasone (Lo-Dex) in patients $(n=38)$ with relapsed/refractory MM who had failed prior therapies, including lenalidomide or bortezomib. Prior therapies in enrolled patients included lenalidomide, bortezomib and dexamethasone (all patients), thalidomide (79\%) and autologous stem cell transplantation (66\%). In the Phase I portion of the study, patients were given escalating doses of pomalidomide $(2,3,4$ or $5 \mathrm{mg})$. The design of this trial allowed the addition of weekly dexamethasone $(40 \mathrm{mg})$ in patients who either developed PD while on pomalidomide monotherapy or did not show response after four cycles. Once the MTD was defined as $4 \mathrm{mg}$, patients were randomized into the phase II part of the study comparing pomalidomide and Lo-Dex versus pomalidomide monotherapy. Two hundred and twentyone patients, with a median age of 63 years and relatively comparable baseline characteristics, were enrolled into two arms. Patients recruited to the pomalidomide monotherapy arm were given Lo-Dex ( $40 \mathrm{mg} /$ week) upon disease progression. Delineating PFS was the primary endpoint. Across both cohorts, a median of five cycles were received by patients; with 34 and $13 \%$ of patients demonstrating a response of $\geqslant P R$ in the pomalidomide plus Lo-Dex arm and pomalidomide monotherapy arms, respectively. ${ }^{41}$ The most notable adverse event was a DLT of grade 4 neutropenia. The updated results for this study were reported by $\mathrm{Vij}$ et al., $^{42}$ showing the ORR in the pomalidomide alone arm to be $9 \%$, whereas the pomalidomide plus Lo-Dex arm achieved an ORR of $30 \%$. The ORR distribution in the pomalidomide plus Lo-Dex cohort was $25 \%$ in lenalidomiderefractory, 29\% in bortezomib-refractory, $28 \%$ in dual (lenalidomide and bortezomib)-refractory and $34 \%$ in dual refractory plus autologous stem cell transplantation patients. The median PFS was an additional 1.3 months in the combined arm as compared with the pomalidomide alone arm (3.8 months versus 2.5 months, respectively; HR $0.73, P=0.037$ ). The median OS in both arms, 13.6 months in pomalidomide-alone arm and 14.4 months in the combined arm (HR 0.85, $P=0.449$ ), was considerably longer than the median OS for patients with PD as best response (4.6 months).

Leleu et al. ${ }^{43}$ conducted a study (IFM 2009-02) comparing two different dosing regimens of pomalidomide and Lo-Dex in dual refractory MM patients. Eighty-four patients refractory to at least two cycles of bortezomib and lenalidomide, and who had received $\geqslant 1$ prior therapy, were divided into two arms. Forty-three patients in arm A were given $4 \mathrm{mg}$ of pomalidomde for 21 days in a 28-day cycle. Forty-one patients in arm $B$ received $4 \mathrm{mg}$ of pomalidomide every day of the 28-day cycle. In both arms, patients received $40 \mathrm{mg}$ of dexamethasone on days 1, 8, 15 and 22 of the cycle. The median age was 60 years and the median number of prior lines of therapy was five. The ORR was $34.9 \%$ in arm A and $34.1 \%$ in arm B. The median PFS was 6.3 months for both arms. The median duration of response was reported as 11.4 and 7.9 months in arm $A$ and arm B, respectively. The incidence of adverse effects was similar in both groups ( $\geqslant$ grade 3 events of 23.5 versus $26 \%$ ), whereas hematological side effects were less prominent in arm A. No cases of DVT or neuropathy were reported. The goal of the final analysis of this study was to ascertain prolonged OS, which has been reported to be 14.9 months; similar in both arms. As compared with the historical control study, an overall improvement in OS was demonstrated along with a median time to progression determined to be at 5 months. ${ }^{44}$

The NIMBUS study, a Phase III trial comparing the efficacy and safety of pomalidomide and Lo-Dex versus high-dose dexamethasone (HiDex) in dual refractory MM patients was recently reported by Dimopoulos et al. $^{45}$ In this study, a total of 455 patients were randomized 2:1 to receive Pomalidomide + Lo-Dex (arm A) or HiDex alone (arm B), respectively. The patients had received a median of five prior lines of treatments and had progressed on 
two or more consecutive cycles of lenalidomide or bortezomib (and in combination), with $72 \%$ of patients being refractory to both agents. Arm A received $4 \mathrm{mg}$ pomalidomide on days 1-21 and $40 \mathrm{mg}$ of dexamethasone on days $1,8,15$ and 22 of a 28-day cycle. HiDex treatment in arm B comprised $40 \mathrm{mg}$ of dexamethasone on days $1-4,9-12$ and $17-20$ of a 28 -day cycle. The primary endpoint was PFS with a median follow-up time of 18 months. PFS for Pomalidomide + Lo-Dex was reported as 15.7 weeks, which had substantially surpassed the PFS of 8 weeks with HiDex treatment (HR 0.45, $P<0.001)$. Even the OS (interim analysis) with Pomalidomide + Lo-Dex was considerably longer than with HiDex alone (median not reached versus 34 weeks, respectively, 134 events, HR 0.53, $P<0.001$ ). Neutropenia (grade $3 / 4$ ) was higher in arm A (42\%) compared with that in arm B (15\%), with other adverse effects being relatively similar between the two arms.

In addition to its pairing with dexamethasone, pomalidomide therapy has been examined in combination with other novel agents as well. Shah et al. ${ }^{46}$ conducted a Phase $1 /$ II trial of carfilzomib and pomalidomide with dexamethasone (Car-Pom-d) in relapsed/refractory MM patients. Interim results from this study indicate an enrollment of 32 heavily pre-treated patients (median number of prior regimens is six) who are lenalidomide-refractory with prior bortezomib exposure along with some patients who had received prior transplant and carry poor-risk cytogenetics [del (17p)]. Determination of MTD was mandated as the primary endpoint and was established as $4 \mathrm{mg}$ for pomalidomide, $40 \mathrm{mg}$ for dexamethasone, $40 \mathrm{mg}$ with carfilzomib being dosed at two levels; $20 / 27 \mathrm{mg} / \mathrm{m}^{2}$ (dose level 1) and $20 / 36 \mathrm{mg} / \mathrm{m}^{2}$ (dose level 2). A high response rate of 2 VGPR, 7 PR and $M R \geqslant 56 \%$ was presented for 27/32 enrolled patients. A larger Phase II cohort has started enrolling patients, and results are eagerly anticipated.

Researchers at Weill Cornell University (Rossi et al. ${ }^{47}$ ) reported a single-institution, Phase II study of clarithromycin, pomalidomide and dexamethasone (ClaPD) in relapsed/refractory MM. A total of 73 patients with lenalidomide-refractory MM who had received three or more prior lines of therapy were recruited in this trial. Patients received $500 \mathrm{mg}$ of clarithromycin twice daily, $40 \mathrm{mg}$ dexamethasone weekly and $4 \mathrm{mg}$ of pomalidomide daily on days 1-21 of a 28-day cycle. Data for 66 patients was recently presented with the ORR being $56 \%$ and a median PFS of 5 months; this is comparable to other regimens used in advanced MM patients. The ORR and PFS were similar irrespective of previous treatment regimens. The adverse effects profile was favorable with only two patients (one patient with fatigue and another with muscular weakness) experiencing grade $\geqslant 3$ toxicities.

Pomalidomide has also been investigated with more traditional chemotherapeutics. In a Phase I/II study, it was examined in combination with cyclophosphamide and prednisone (PCP) in relapsed/refractory MM patients. ${ }^{48}$ Palumbo et al. report on the interim results of 52 patients. When used in combination with cyclophosphamide and prednisone, the MTD for pomalidomide was determined to be $2.5 \mathrm{mg}$. The ORR was reported as $79 \%$, and the 1 -year PFS was 52\%. The incidence of grade 4 neutropenia was $13 \%$ and one patient had a venous thrombo-embolic event while being on prophylaxis.

The most common (all grades $\geqslant 10 \%$ ) hematologic and nonhematologic pomalidomide-related toxicities as observed in most patients are listed in Table 2.

\section{CONCLUSION}

IMiDs are a novel group of anticancer agents that have revolutionized the treatment landscape for patients with MM and other hematologic malignancies. Mechanistically, they exert their effect on elements of the tumor microenvironment by modulation of tumor-supporting cytokines, immune effector cell activation and engaging support from non-immune host cells; however, the exact mechanisms of action of IMiDs remain unclear. ${ }^{49}$ It is likely that the combination of these properties directs the antitumor effects specific to the disease being treated, with some of these effects being more prominent than others and differentially expressed based on the cell on which the molecule is acting. Over the last decade, collective experience has demonstrated through several phase III studies that immunotherapy with small molecules such as IMiDs is one of the most potent and consistent strategies to achieve durable disease control along with extending PFS and OS in

Table 2. Most commonly observed $(\geqslant 10 \%)$ pomalidomide-related adverse events

\begin{tabular}{|c|c|c|c|}
\hline \multirow[t]{2}{*}{ Study } & \multirow[t]{2}{*}{ Regimen } & \multicolumn{2}{|c|}{ Adverse event } \\
\hline & & Hematologic & Non-hematologic \\
\hline Schey et al. $^{34}$ & Pom & Neutropenia & Skin toxicity, GI toxicity, neuropathy, edema, DVI \\
\hline Streetly et al. ${ }^{35}$ & Pom + dex & Neutropenia, thrombocytopenia & $\begin{array}{l}\text { Rash, constipation, edema, abdominal bloating, } \\
\text { pyrexia, light-headedness, tremor }\end{array}$ \\
\hline Richardson et al. ${ }^{40,41}$ & Pom + Dex & Neutropenia, anemia, thrombocytopenia, DVT & Infection, diarrhea, musculoskeletal pain, fatigue \\
\hline Lacy et al. ${ }^{36}$ & Pom + Dex & Anemialeukopenia, thrombocytopenia & $\begin{array}{l}\text { Fatigue, nausea, diarrhea/constipation, } \\
\text { pneumonia, hyperglycemia, insomnia/agitation, } \\
\text { peripheral neuropathy }\end{array}$ \\
\hline Lacy et $a .^{37, a}$ & Pom + Dex & $\begin{array}{l}\text { Neutropenia, anemia, ,lymphopenia, } \\
\text { thrombocytopenia, leukopenia }\end{array}$ & $\begin{array}{l}\text { Hyperglycemia, peripheral neuropathy, fatigue } \\
\text { anorexia, diarrea, nausea }\end{array}$ \\
\hline Leleu et al. & Pom + Dex & Myelosuppression & NR \\
\hline \multirow[t]{2}{*}{ Lacy et al. ${ }^{39, b}$} & $\begin{array}{l}\text { Pom }+ \text { Dex Cohort A } \\
\text { (Pom } 2 \mathrm{mg} \text { ) }\end{array}$ & $\begin{array}{l}\text { Neutropenia, anemia, leukopenia, } \\
\text { lymphopenia, thrombocytopenia }\end{array}$ & $\begin{array}{l}\text { Pneumonia, peripheral neuropathy, renal failure, } \\
\text { atrial fibrillation ( } 9 \%) \text {, hyperglycemia }(9 \%) \text {, } \\
\text { fatigue, anorexia diarrhea, nausea/vomiting }\end{array}$ \\
\hline & $\begin{array}{l}\text { Cohort B } \\
\text { (Pom } 4 \mathrm{mg} \text { ) }\end{array}$ & $\begin{array}{l}\text { Anemia, leukopenia, lymphopenia, } \\
\text { neutropenia, thrombocytopenia }\end{array}$ & $\begin{array}{l}\text { Febrile neutropenia, peripheral neuropathy, } \\
\text { fatigue anorexia, diarrhea, nausea }\end{array}$ \\
\hline \multirow[t]{2}{*}{ Dimopoulos et al. ${ }^{45}$} & Arm A (Pom + dex) & Neutropenia, thrombocytopenia & Infections \\
\hline & Arm B (HiDex) & Neutropenia, thrombocytopenia & Infections \\
\hline Shah et al. ${ }^{46, c}$ & Carf + Pom + Dex & Anemia, thrombocytopenia & Fatigue, pneumonia, dyspnea, rash \\
\hline Rossi et al. ${ }^{47, c}$ & Clar + Pom + Dex & None & Toxicities, $<2 \%$ \\
\hline Palumbo et al. ${ }^{48, c}$ & Cyclo + Pom + Pred & Neutropenia & Toxicities, <9\% \\
\hline
\end{tabular}

Abbreviations: Carf, carfilzomib; Clar, clarithromycin; Cyclo, cyclophosphamide; Dex, dexamethasone; Pom, pomalidomide; Pred, prednisone. ${ }^{\text {aStudy }}$ was conducted in lenalidomide-refractory patients; ${ }^{b}$ Study was conducted in lenalidomide- and bortezomib-refractory patients; ' ${ }^{C}$ Study is ongoing. 
myeloma patients. Currently the IMiDs class of drugs includes three agents: thalidomide, lenalidomide and the forthcoming pomalidomide. Interestingly, their clinical effectiveness displays unique variability allocating disease responsiveness to each one of the agents. It is known that patients responding to thalidomide upon progression can be salvaged with lenalidomide, ${ }^{50}$ and those who failed lenalidomide have now been shown to secure clinical remission with pomalidomide. Intriguingly, in a subset analysis of patient who have failed pomalidomide therapy ${ }^{36,37}$ and subsequently received a lenalidomide-based salvage treatment regimen, an ORR of $29 \%$ was achieved. ${ }^{51}$ Taken together, these observations are important as they expose the critical and continuous role of immune therapy during the course of disease along with substantiating that a failed response to one of the IMiDs molecules does not obviate utility of the other agents in this class of therapeutics.

Pomalidomide is the newest of the IMiD molecules and preclinical data support its more powerful inhibitory effect on target cytokines compared with its sister drugs, suggesting improved potency. This observation has proved useful in the clinical setting as noted in the results from ongoing clinical trials in heavily pre-treated $\mathrm{MM}$ patients, including those resistant to lenalidomide or bortezomib (or both). Similar to prior experience with other IMiDs, the response to pomalidomide appears higher when combined with dexamethasone. The addition of a third agent (carfilzomib, clarithromycin or cyclophosphamide) to this combination has demonstrated improved efficacy in the ORR with an acceptable toxicity profile. It is important to note that while responses to pomalidomide therapy are encouraging in the relapsed or relapse/refractory patient population, the incidence of clinical responses become lower as the number of prior therapies increased and/or as patients who were enrolled on the aforementioned trials had previously acquired resistance to lenalidomide or bortezomib. Furthermore, there was a lack of any clinical trial demonstrating $C R$ among patients treated. This may be an important limitation of pomalidomide therapy, considering that the depth of anti-myeloma response is clinically important and several of the current treatment regimens are able to deliver higher incidence of CR rates, even in the relapsed or refractory patient population. ${ }^{52}$ As all the pomalidomide trials to date have been conducted in advanced-stage myeloma patients, future studies to examine this agent's role in the up-front setting of treatment-naive patients are warranted. In the absence of such data, development of an appropriate strategy to sequence the IMiD molecules for therapeutic efficacy and biologic response indeed presents a challenge. With the use of novel therapies, the OS of MM patients has improved significantly. ${ }^{53,54}$ This, however, has been associated with an increased incidence of extramedullary myelomatous disease (EMD) development in these patients, and the role of IMiDs and their efficacy has been called into question. ${ }^{55,56}$ As the survival of MM patients increases due to the high anti-MM activity of novel agents, an increased probability of selection for fitter MM clones that are less reliant on the bone marrow milieu for their survival also emerges. In a subset analysis of 174 patients, the incidence of treatment-emergent EMD was $7.5 \%$. Importantly, pomalidomide plus Lo-Dex therapy was capable of eliciting a $\sim 30 \%$ response and $\geqslant 50 \%$ reduction of the EMD component. ${ }^{57}$

Table 3. Ongoing clinical trials of pomalidomide in multiple myeloma

Study to determine the maximum tolerated dose for the combination of pomalidomide, bortezomib and low-dose dexamethasone in subjects with relapsed or refractory multiple myeloma

Study of pomalidomide to evaluate the pharmacokinetics and safety for patients with multiple myeloma and impaired renal function (POM renal)

MTD, safety, and efficacy of CC-4047 alone with dexamethasone in patients with relapsed and refractory multiple myeloma

Japanese Phase 1 study to evaluate tolerated dose, safety, and efficacy of pomalidomide in patients with refractory or relapsed and refractory multiple myeloma

Pomalidomide, cyclophosphamide and prednisone (PCP) in patients with multiple myeloma (MM) relapsed and/or refractory to lenalidomide

Pomalidomide, bortezomib, and dexamethasone in treating patients with relapsed or refractory multiple myeloma Pomalidomide in combination with high dose dexamethasone and oral cyclophosphamide

A Safety and efficacy study of carfilzomib and pomalidomide with dexamethasone in patients with relapsed or refractory multiple myeloma

A Phase $1 / 2$ study of pomalidomide, dexamethasone and pegylated liposomal doxorubicin for patients with relapsed/refractory multiple myeloma

Bendamustine + pomalidomide + dex in R/R multiple myeloma

Carfilzomib, pomalidomide, and dexamethasone in treating patients with relapsed or refractory multiple myeloma CC-4047 and dexamethasone in treating patients with relapsed or refractory multiple myeloma or amyloidosis IFM2009-02-pomalidomide and dexamethasone in myeloma

Study of decadron, biaxin, and pomalidomide in relapsed/refractory myeloma

Pomalidomide in gene expression profiling (gep)-defined high-risk multiple myeloma

Continuous versus intermittent dosing regimens for pomalidomide in relapsed/refractory multiple myeloma

Pomalidomide (POM) in combination with low dose dexamethasone (LD-Dex) in patients with relapsed or refractory multiple myeloma (PEXIUS)

Autologous stem cell transplant with pomalidomide (CC-4047) maintenance versus continuous pomalidomide/ dexamethasone salvage therapy in relapsed or refractory multiple myeloma

Pomalidomide and dexamethasone effects in multiple myeloma patients with Del 17p or t (4;14) (IFM2010-02)

Study to compare efficacy and safety of pomalidomide in combination with low-dose dexamethasone versus highdose dexamethasone in subjects with refractory or relapsed and refractory multiple myeloma (NIMBUS)

Study to evaluate the safety and efficacy of pomalidomide monotherapy in subjects with refractory or relapsed refractory multiple myeloma

Evaluation of safety of pomalidomide in combination with dexamethasone (low dose) in patients with refractory or relapsed and refractory multiple myeloma (STRATUS)

Safety and efficacy of pomalidomide, bortezomib and low-dose dexamethasone in subjects with relapsed or refractory multiple myeloma (OPTIMISMM)
Identifier

NCT01497093

NCT01575925

NCT00833833

NCT01568294

NCT01166113

NCT01212952

NCT01432600

NCT01464034

NCT01541332

NCT01754402

NCT01665794

NCT00558896

NCT01053949

NCT01159574

NCT01177735

NCT01319422

NCT01632826

NCT01745588

NCT01745640

NCT01311687

NCT01324947

Study stage

Phase I

Phase I

Phase I

Phase I

Phase I/II

Phase 1/II

Phase I/II

Phase I/II

Phase I/II

Phase I/II Phase I/II

Phase Ib/II

Phase II

Phase II

Phase II

Phase II

Phase II

Phase II

Phase II

Phase III

Phase III

NCT01712789

Phase III

NCT01734928

Phase III 
In summary, pomalidomide is a promising anti-myeloma agent that has the ability to induce remission in patients with heavily pre-treated MM. The toxicity profile is predictable and manageable. Development of pomalidomide marks an important era for patients with $\mathrm{MM}$, as it brings an important active therapeutic option for the management of this disease. It has been a distinct addition to the consortium of anti-myeloma drugs in relapsed and refractory MM. Table 3 summarizes some of the ongoing clinical trials testing pomalidomide in patients with MM. Their results are eagerly anticipated and will further define the role of pomalidomide in MM

\section{CONFLICT OF INTEREST}

ML receives funding from Celgene. SK is a paid consultant for Merck, an unpaid consultant for Celgene and Millennium, and receives funding from Celgene, Millennium and Novartis. AD receives funding from Millenium, Celgene and Pfizer. ACK is an unpaid consultant for Celgene. The remaining authors declare no conflict of interest.

\section{ACKNOWLEDGEMENTS}

We would like to thank Ms Victoria L Jackson, MLIS, and Ms Kelly Viola (Academic and Research Support, Mayo Clinic, Jacksonville, FL, USA) for their editorial assistance in the preparation of this manuscript.

\section{REFERENCES}

1 Singhal S, Mehta J, Desikan R, Ayers D, Roberson P, Eddlemon P et al. Antitumor activity of thalidomide in refractory multiple myeloma. $N$ Engl J Med 1999; 341: 1565-1571.

2 Kotla V, Goel S, Nischal S, Heuck C, Vivek K, Das B et al. Mechanism of action of lenalidomide in hematological malignancies. J Hematol Oncol 2009; 2: 36.

3 Swaika A, Paulus A, Chanan-Khan A. Pomalidomide in cancer therapy (pp. 253-265). In Chanan-Khan AA, Immunomodulating Drugs for the Treatment of Cancer. Philadelphia: Lippincott, Williams \& Wilkins.

4 Chanan-Khan A, Miller KC, Musial L, Lawrence D, Padmanabhan S, Takeshita K et al. Clinical efficacy of lenalidomide in patients with relapsed or refractory chronic lymphocytic leukemia: results of a phase II study. J Clin Oncol 2006; 24: 5343-5349.

5 Muller GW, Chen R, Huang SY, Corral LG, Wong LM, Patterson RT et al. Amino-substituted thalidomide analogs: potent inhibitors of TNF-alpha production. Bioorg Med Chem Lett 1999; 9: 1625-1630.

6 Bartlett JB, Dredge K, Dalgleish AG. The evolution of thalidomide and its IMiD derivatives as anticancer agents. Nat Rev Cancer 2004; 4: 314-322.

7 Quach H, Ritchie D, Stewart AK, Neeson P, Harrison S, Smyth MJ et al. Mechanism of action of immunomodulatory drugs (IMiDS) in multiple myeloma. Leukemia 2010; 24: 22-32

8 Corral LG, Haslett PA, Muller GW, Chen R, Wong LM, Ocampo CJ et al. Differential cytokine modulation and $\mathrm{T}$ cell activation by two distinct classes of thalidomide analogues that are potent inhibitors of TNF-alpha. J Immunol 1999; 163: 380-386.

9 Davies FE, Raje N, Hideshima T, Lentzsch S, Young G, Tai YT et al. Thalidomide and immunomodulatory derivatives augment natural killer cell cytotoxicity in multiple myeloma. Blood 2001; 98: 210-216.

10 Payvandi F, Wu L, Naziruddin SD, Haley M, Parton A, Schafer PH et al. Immunomodulatory drugs (IMiDs) increase the production of IL-2 from stimulated T cells by increasing PKC-theta activation and enhancing the DNA-binding activity of AP-1 but not NF-kappaB, OCT-1, or NF-AT. J Interferon Cytokine Res 2005; 25: 604-616.

11 Schafer PH, Gandhi AK, Loveland MA, Chen RS, Man HW, Schnetkamp PP et al. Enhancement of cytokine production and AP-1 transcriptional activity in T cells by thalidomide-related immunomodulatory drugs. J Pharmacol Exp Ther 2003; 305: 1222-1232.

12 Hayashi T, Hideshima T, Akiyama M, Podar K, Yasui $\mathrm{H}$, Raje $\mathrm{N}$ et al. Molecular mechanisms whereby immunomodulatory drugs activate natural killer cells: clinical application. Br J Haematol 2005; 128: 192-203.

13 Hernandez-llizaliturri FJ, Reddy N, Holkova B, Ottman E, Czuczman MS Immunomodulatory drug CC-5013 or CC-4047 and rituximab enhance antitumor activity in a severe combined immunodeficient mouse lymphoma model. Clin Cancer Res 2005; 11: 5984-5992.

14 Dredge K, Marriott JB, Todryk SM, Muller GW, Chen R, Stirling DI et al. Protective antitumor immunity induced by a costimulatory thalidomide analog in conjunction with whole tumor cell vaccination is mediated by increased Th1-type immunity. J Immunol 2002; 168: 4914-4919.

15 Galustian C, Meyer B, Labarthe MC, Dredge K, Klaschka D, Henry J et al. The anti-cancer agents lenalidomide and pomalidomide inhibit the proliferation and function of T regulatory cells. Cancer Immunol Immunother 2009; 58: 1033-1045.

$16 \mathrm{Xu} \mathrm{Y,} \mathrm{Li} \mathrm{J,} \mathrm{Ferguson} \mathrm{GD,} \mathrm{Mercurio} \mathrm{F,} \mathrm{Khambatta} \mathrm{G,} \mathrm{Morrison} \mathrm{L} \mathrm{et} \mathrm{al.}$ Immunomodulatory drugs reorganize cytoskeleton by modulating Rho GTPases. Blood 2009; 114: 338-345.

17 Anderson G, Gries M, Kurihara N, Honjo T, Anderson J, Donnenberg V et al. Thalidomide derivative CC-4047 inhibits osteoclast formation by down-regulation of PU.1. Blood 2006; 107: 3098-3105.

18 Breitkreutz I, Raab MS, Vallet S, Hideshima T, Raje N, Mitsiades C et al. Lenalidomide inhibits osteoclastogenesis, survival factors and bone-remodeling markers in multiple myeloma. Leukemia 2008; 22: 1925-1932.

19 Hideshima T, Chauhan D, Shima Y, Raje N, Davies FE, Tai YT et al. Thalidomide and its analogs overcome drug resistance of human multiple myeloma cells to conventional therapy. Blood 2000; 96: 2943-2950.

20 Reddy N, Hernandez-llizaliturri FJ, Deeb G, Roth M, Vaughn M, Knight J et al. Immunomodulatory drugs stimulate natural killer-cell function, alter cytokine production by dendritic cells, and inhibit angiogenesis enhancing the anti-tumour activity of rituximab in vivo. Br J Haematol 2008; 140: 36-45.

21 Lu L, Payvandi F, Wu L, Zhang LH, Hariri RJ, Man HW et al. The anti-cancer drug lenalidomide inhibits angiogenesis and metastasis via multiple inhibitory effects on endothelial cell function in normoxic and hypoxic conditions. Microvasc Res 2009; 77: 78-86.

22 Ladetto M, Vallet S, Trojan A, Dell'Aquila M, Monitillo L, Rosato R et al. Cyclooxygenase-2 (COX-2) is frequently expressed in multiple myeloma and is an independent predictor of poor outcome. Blood 2005; 105: 4784-4791.

23 Prince HM, Mileshkin L, Roberts A, Ganju V, Underhill C, Catalano J et al. A multicenter phase II trial of thalidomide and celecoxib for patients with relapsed and refractory multiple myeloma. Clin Cancer Res 2005; 11: 5504-5514.

24 Ferguson GD, Jensen-Pergakes K, Wilkey C, Jhaveri U, Richard N, Verhelle D et al. Immunomodulatory drug CC-4047 is a cell-type and stimulus-selective transcriptional inhibitor of cyclooxygenase 2. J Clin Immunol 2007; 27: 210-220.

25 Escoubet-Lozach L, Lin IL, Jensen-Pergakes K, Brady HA, Gandhi AK, Schafer PH et al. Pomalidomide and lenalidomide induce p21 WAF-1 expression in both lymphoma and multiple myeloma through a LSD1-mediated epigenetic mechanism. Cancer Res 2009; 69: 7347-7356.

26 Mitsiades N, Mitsiades CS, Poulaki V, Chauhan D, Richardson PG, Hideshima T et al. Apoptotic signaling induced by immunomodulatory thalidomide analogs in human multiple myeloma cells: therapeutic implications. Blood 2002; 99: 4525-4530.

27 Li S, Pal R, Monaghan SA, Schafer P, Ouyang H, Mapara M et al. IMiD immunomodulatory compounds block $\mathrm{C} / \mathrm{EBP}$ \{beta\} translation through elF4E down-regulation resulting in inhibition of MM. Blood 2011; 117: 5157-5165.

28 Ito $\mathrm{T}$, Ando $\mathrm{H}$, Suzuki T, Ogura T, Hotta K, Imamura $\mathrm{Y}$ et al. Identification of a primary target of thalidomide teratogenicity. Science 2010; 327: 1345-1350.

29 Lopez-Girona A, Mendy D, Ito T, Miller K, Gandhi AK, Kang J et al. Cereblon is a direct protein target for immunomodulatory and antiproliferative activities of lenalidomide and pomalidomide. Leukemia 2012; 26: 2326-2335.

30 Yang $Y$, Shaffer 3rd AL, Emre NC, Ceribelli M, Zhang M, Wright G et al. Exploiting synthetic lethality for the therapy of $A B C$ diffuse large $B$ cell lymphoma. Cancer Cell 2012; 21: 723-737.

31 Zhu YX, Braggio E, Shi CX, Bruins LA, Schmidt JE, Van Wier S et al. Cereblon expression is required for the antimyeloma activity of lenalidomide and pomalidomide. Blood 2011; 118: 4771-479.

32 Zhu YX, Kortuem KM, Stewart AK. Molecular mechanism of action of immunemodulatory drugs thalidomide, lenalidomide and pomalidomide in multiple myeloma. Leuk Lymphoma 2012; 54: 683-687.

33 Schuster SR, Kortuem KM, Zhu YX, Braggio E, Shi C-X, Bruins L et al. Cereblon expression predicts response, progression free and overall survival after pomalidomide and dexamethasone therapy in multiple myeloma. ASH Annual Meeting Abstracts 2012; 120: 194.

34 Schey SA, Fields P, Bartlett JB, Clarke IA, Ashan G, Knight RD et al. Phase I study of an immunomodulatory thalidomide analog, CC-4047, in relapsed or refractory multiple myeloma. J Clin Oncol 2004; 22: 3269-3276.

35 Streetly MJ, Gyertson K, Daniel Y, Zeldis JB, Kazmi M, Schey SA. Alternate day pomalidomide retains anti-myeloma effect with reduced adverse events and evidence of in vivo immunomodulation. Br J Haematol 2008; 141: 41-51.

36 Lacy MQ, Hayman SR, Gertz MA, Dispenzieri A, Buadi F, Kumar S et al. Pomalidomide (CC4047) plus low-dose dexamethasone as therapy for relapsed multiple myeloma. J Clin Oncol 2009; 27: 5008-5014.

37 Lacy MQ, Hayman SR, Gertz MA, Short KD, Dispenzieri A, Kumar S et al. Pomalidomide (CC4047) plus low dose dexamethasone (Pom/dex) is active and well tolerated in lenalidomide refractory multiple myeloma (MM). Leukemia 2010; 24: 1934-1939. 
38 Kumar SK, Lee JH, Lahuerta JJ, Morgan G, Richardson PG, Crowley J et al. Risk of progression and survival in multiple myeloma relapsing after therapy with IMiDs and bortezomib: a multicenter international myeloma working group study. Leukemia 2012; 26: 149-157.

39 Lacy MQ, Allred JB, Gertz MA, Hayman SR, Short KD, Buadi F et al. Pomalidomide plus low-dose dexamethasone in myeloma refractory to both bortezomib and lenalidomide: comparison of 2 dosing strategies in dual-refractory disease. Blood 2011; 118: 2970-2975.

40 Richardson PG, Siegel D, Baz R, Kelley SL, Munshi NC, Sullivan D et al. A phase 1/2 multi-center, randomized, open label dose escalation study to determine the maximum tolerated dose, safety, and efficacy of pomalidomide alone or in combination with low-dose dexamethasone in patients with relapsed and refractory multiple myeloma who have received prior treatment that includes lenalidomide and bortezomib. ASH Annual Meeting Abstracts 2010; 116: 864.

41 Richardson PG, Siegel DS, Vij R, Hofmeister CC, Jagannath S, Chen C et al. Randomized, open label phase 1/2 study of pomalidomide (POM) alone or in combination with low-dose dexamethasone (LoDex) in patients (Pts) with relapsed and refractory multiple myeloma who have received prior treatment that includes lenalidomide (LEN) and bortezomib (BORT) phase 2 results. ASH Annual Meeting Abstracts 2011; 118: 634.

42 Vij RR, Jagannath PG, Siegel S, Baz DS, Srinivasan RC, Larkins S et al. (POM) with or without low-dose dexamethasone (LoDEX) in patients (pts) with relapsed/ refractory multiple myeloma (RRMM): outcomes in pts refractory to lenalidomide (LEN) and/or bortezomib (BORT). J Clin Oncol 2012; 30: (Abstract 8016).

43 Leleu X, Attal M, Moreau P, Duhamel A, Fermand JP, Michalet M et al. Phase 2 study of 2 modalities of pomalidomide (CC4047) plus low-dose dexamethasone as therapy for relapsed multiple myeloma. IFM 2009-02. ASH Annual Meeting Abstracts 2010; 116: 859 .

44 Leleu X, Roussel M, Arnulf B, Moreau P, Traulle C, Michalet M et al. Prolonged overall survival with pomalidomide and dexamethasone in myeloma characterized with end stage disease. ASH Annual Meeting Abstracts 2012; 120: 2961.

45 Dimopoulos MA, Lacy MQ, Moreau P, Weisel KC, Song KW, Delforge M et al. Pomalidomide in Combination with low-dose dexamethasone: demonstrates significant progression free survival and overall survival advantage, in relapsed/ refractory MM: a phase 3, multicenter, randomized open-label study. ASH Annual Meeting Abstracts 2012; 120: LBA- 6

46 Shah JJ, Stadtmauer EA, Abonour R, Cohen AD, Bensinger WI, Gasparetto C et al. A multi-center phase $\mathrm{I} / \mathrm{II}$ trial of carfilzomib and pomalidomide with dexamethasone (Car-Pom-d) in patients with relapsed/refractory multiple myeloma. ASH Annual Meeting Abstracts 2012; 120: 74.
47 Rossi AC, Mark TM, Rodriguez M, Shah M, Quinn R, Pearse R et al. Clarithromycin pomalidomide, and dexamethasone (ClaPD) in relapsed or refractory multiple myeloma. J Clin Oncol 2012; 30: (Abstract 8036).

48 Palumbo A, Larocca A, Montefusco V, Rossi D, Carella AM, Mina R et al. Pomalidomide cyclophosphamide and prednisone (PCP) treatment for relapsed/refractory multiple myeloma. ASH Annual Meeting Abstracts 2012; 120: 446.

49 Chanan-Khan AA, Cheson BD. Lenalidomide for the treatment of B-cell malignancies. J Clin Oncol 2008; 26: 1544-1552.

50 Wang M, Knight R, Dimopoulos M, Siegel D, Rajkumar SV, Facon $T$ et al. Lenalidomide in Combination with dexamethasone was more effective than dexamethasone in patients who have received prior thalidomide for relapsed or refractory multiple myeloma. ASH Annual Meeting Abstracts 2006; 108: 3553.

51 Sinha S, Lacy M, Mikhael J, Hayman S, Buadi F, Detweiler-Short K et al. Response to salvage therapies and outcome in patients with multiple myeloma relapsing after pomalidomide therapy. Leukemia 2012; 26: 839-841.

52 Chanan-Khan AA, Giralt S. Importance of achieving a complete response in multiple myeloma, and the impact of novel agents. Journal Clin Oncol 2010; 28 2612-2624.

53 Kumar SK, Rajkumar SV, Dispenzieri A, Lacy MQ, Hayman SR, Buadi FK et al. Improved survival in multiple myeloma and the impact of novel therapies. Blood 2008; 111: 2516-2520.

54 Kastritis E, Zervas K, Symeonidis A, Terpos E, Delimbassi S, Anagnostopoulos N et al. Improved survival of patients with multiple myeloma after the introduction of novel agents and the applicability of the International Staging System (ISS): an analysis of the Greek Myeloma Study Group (GMSG). Leukemia 2009; 23: 1152-1157.

55 Rosinol L, Cibeira MT, Blade J, Esteve J, Aymerich M, Rozman M et al. Extramedullary multiple myeloma escapes the effect of thalidomide. Haematologica 2004; 89: 832-836.

56 Varettoni M, Corso A, Pica G, Mangiacavalli S, Pascutto C, Lazzarino M. Incidence, presenting features and outcome of extramedullary disease in multiple myeloma: a longitudinal study on 1003 consecutive patients. Ann Oncol 2010; 21: 325-3230.

57 Short KD, Rajkumar SV, Larson D, Buadi F, Hayman S, Dispenzieri A et al. Incidence of extramedullary disease in patients with multiple myeloma in the era of novel therapy, and the activity of pomalidomide on extramedullary myeloma. Leukemia 2011; 25: 906-908.

$(\$$ This work is licensed under a Creative Commons AttributionCy NC No NonCommercial-NoDerivs 3.0 Unported License. To view a copy of this license, visit http://creativecommons.org/licenses/by-nc-nd/3.0/ 